\title{
Lessons to Learn from Rare Inborn Errors of Metabolism
}

This issue contains a most interesting and comprehensive case report by D. Zafeiriou and coauthors [13] on one of the very rare inborn errors of metabolism - ethylmalonic encephalopathy (EE). Since the first description in 1991 by Burlina et al. [3] only about 35 patients, mostly of Mediterranean descent, have been described. Knowledge on very rare inborn errors of metabolism may not only contribute to our every day clearup rate of unexplained cases, but may also improve our understanding of the molecular pathomechanisms involved in CNS disease. The delineation of ethylmalonic encephalopathy has been intriguing and despite the identification of the underlying gene defect by Tiranti et al. in 2004 [10], the molecular mechanisms are only partly understood today.

EE is characterized by an early onset progressive encephalopathy and vasculopathy with chronic diarrhea, recurrent petechiae and orthostatic acrocyanosis, leading to death in early infancy or childhood. Although the combination of these clinical features seems to be fairly constant, there is variability in the disease onset and severity, recognized with the number of reports published. In some patients there may be a period of near normal early psychomotor development, while others have complete lack of motor development and social interaction. Diarrhea in EE usually starts after weaning and frequent watery stools have led to failure to thrive in some cases. Petechiae may occur spontaneously or with intercurrent illness and are easily provoked by minimal pressure. Diarrhea and petechiae may precede the onset of neurological symptoms and some patients have undergone extended hematological or gastrointestinal work-up before the developmental delay had become more prominent $[4,6]$. Patients affected by EE all suffer from some degree of muscle hypotonia followed by spasticity and dystonia $[6,7,9]$ but independent walking may be achieved in milder cases [9]. In those cases with a milder course, cognitive function seems to be initially well preserved, while the more severe cases show profound mental retardation and early-onset seizures. Episodes of metabolic decompensation with severe metabolic acidosis as well as severe nephrotic syndrome have been observed in single patients [1]. Cranial MRI is always abnormal but per se unspecific with bilateral signal changes in the basal

Re: Zafeiriou DI, Augoustides-Savvapoulou P, Haas D, Triantafyllou P, Vargiami E, Tamiolaki M, Gombakis N, Van Coster R, Sewell AC, Vianey-Saban C, Gregersen N: Ethylmalonic Encephalopathy: Clinical and Biochemical Observations ganglia, and white matter involvement with brain atrophy in the more progressed state. Chiari I malformation and tethered cord have been described in two siblings [9] but direct association with the primary defect needs proof in further cases.

Lactic acid elevation and excretion of ethylmalonic acid have been detected as biochemical markers in the first Italian patients [3], but in some patients these have recently been recognized to be of intermittent occurrence along with metabolic decompensation only $[4,8]$. As outlined in the case report by Zafeiriou, the excretion of ethylmalonic acid is non-specific and can also be seen in other inborn errors of metabolism affecting energy production. Nevertheless, the presence of diarrhea and recurrent petechiae clearly distinguish this disorder from phenotypes described in short-chain acyl CoA dehydrogenase deficiency and multiple acyl CoA dehydrogenase deficiency. The source of ethylmalonic acid as well as the accompanying elevation of plasma $C_{4}$ and $C_{5}$ acylcarnitine and acylglycine fractions observed in EE are still unclear. It has been noted early that some patients with EE have mild reduction of their cytochrome $\mathrm{C}$ oxidase activity in muscle tissue [5], but again, no correlation to the excretion of ethylmalonic acid could be established. In order to promote the activity of enzymes involved in the beta-oxidation of fatty acids and in mitochondrial function, Yoon et al. had undertaken a cofactor trial with riboflavine, coenzyme $\mathrm{Q}$ and carnitine in three patients with EE with some clinical improvement, especially with respect to diarrhea, while no consistent change of the laboratory abnormalities could be observed [12].

In 2004 the underlying molecular defect of EE has been detected by Tiranti and co-workers [10]. In a first step a chromosomal region spanning about 130 genes was identified on chromosome $19 q 13$ by homozygosity mapping. Considering the clinical and biochemical findings, genes related to mitochondrial function were then selected within this region by a so-called neighborhood index. This index reflects the similarity of proteins targeted to mitochondria on an RNA level. The gene finally identified as the ethylmalonic encephalopathy ETHE1 gene had previously been found overexpressed in human hepatoma cell cultures and highlights the role of mitochondrial dysfunction not only in inborn errors of metabolism but also in carcinogenesis. Loss of function mutations as well as missense mutations of the ETHE1 gene have since been described in EE patients. 
The propositus described in the case report by Zafeiriou, corresponds to the severe spectrum described in EE. Regarding the clinical, neuroradiological and biochemical findings observed in $\mathrm{EE}$, some patients (especially with intermittent excretion of ethylmalonic acid) may be misdiagnosed as Leighs disease. Aicardi Goutières syndrome may be another differential diagnosis with respect to early onset encephalopathy associated with vasculopathy and acrocyanosis, but in those patients basal ganglia changes represent calcifications, white matter changes are more marked and elevated interferon in CSF would serve as a diagnostic marker. The diagnosis of EE may be extremely difficult before the onset of neurological symptoms but entering the search terms "diarrhea and petechiae" into PubMed would bring up 6 citations out of 87 with this differential diagnosis. The biochemical findings in Zafeiriou's patient diagnosed with EE are typical in the degree of ethylmalonic acid excretion, less so with the isolated elevation of the $C_{4}$ but normal $C_{5}$ fraction of the acylcarnitines, but authors have clearly ruled out SCAD deficiency by enzymology and molecular analysis of the SCAD gene. Analysis of the respiratory chain in muscle of this patient revealed normal activity of cytochrome $\mathrm{C}$ oxidase, while complex II was found to be mildly decreased. The fact that abnormalities of the respiratory chain enzymes are only found in some EE patients, point towards indirect effects by secondary impairement of respiratory chain enzymes, possibly caused by toxic effects of the ethylmalonic acid [2].

The clinical and neuroradiological findings along with the lactic acidosis observed in EE could well be in line with a mitochondrial disorder. The actual hypothesis for the function of the ETHE1 protein is that it acts as a thioesterase on an as yet unknown substrate involved in a novel mitochondrial pathway [11]. It has to be proven if early diagnosis and cofactor treatment can alter the course of this devastating disease.

B. Plecko

References

1 Al-Ajmi MO, Kutty SK. Ethylmalonicaciduria encephalopathy with respiratory failure and nephrotic syndrome. Middle East Journal of Medicine 2005; 3: 1-5
2 Barschak AG, Ferreira G, Andre KR, Schuck PF, Viegas CM, Tonin A, Dutra Filho CS, Wyse AT, Wannmacher CM, Vargas CR, Wajner M. Inhibition of the electron transport chain and creatine kinase activity by ethylmalonic acid in human skeletal muscle. Metab Brain Dis 2006; 21: $11-19$

3 Burlina AB, Dionisi-Vici C, Bennett MJ, Gibson KM, Servidei S, Bertini E, Hale DE, Schmitd-Sommerfeld E, Sabetta G, Zacchello F. A new syndrome with ethylmalonic aciduria and normal fatty acid oxidation in fibroblasts. J Pediatr 1994; 124: 79-86

4 Di Rocco M. Carudo U, Briem E, Rossi A, Allegri A, Buzzi D, Tiranti V. A case of ethylmalonic encephalopathy with atypical clinical and biochemical findings. Mol Gen Metab 2006; 89: 395-397

5 Garavaglia B, Colamaria V, Carrara F, Tonin P, Rimoldi M, Uziel G. Muscle cytochrome $c$ oxidase deficiency in two Italian patients with ethylmalonic aciduria and peculiar phenotype. J Inherit Metab Dis 1994; 17: 301-303

6 Grosso S, Mostardini R, Farnetani MA, Molinelli M, Berardi R, Dionisi-Vici C, Rizzo C, Morgese G, Balestri P. Ethylmalonic encephalopathy. J Neurol 2002; 249: 1446-1450

7 Heberle LC, Tawari AA, Ramadan DG, Ibrahim JK. Ethylmalonic encephalopathy-report of two cases. Brain and Development 2006; 28 : 329-331

8 Merinero B, Perez-Cerda C, Ruiz Sala P, Ferrer I, Garcia MJ, Pardo MM, Belanger Quintana A, Molta JL de la, Martin-Hernandez E, Vianey-Saban C, Bischoff C, Gregersen N, Ugarte M. Persistent increase of plasma butyryl/isobutyrylcarnitine concentrations as markers of SCAD defect and ethylmalonic encephalopathy. J Inherit Metab Dis 2006, short report \#022 online

9 Nowaczyk MJM, Blaser SI, Clarke JTR. Central nervous system malformations in ethylmalonic encephalopathy. Am J Med Genet 1998; 75 : 292-296

10 Tiranti VD, Adarmo R, Briem E, Ferrari G, Mineri R, Lamantea E, Mandel $H$ Balestri P, Garcia Sylva MT, Vollmer B, Rinaldo P, Hahn SH, Leonard J, Rahman S, Dionisi-Vici C, Garavaglia B, Gasparini P, Zeviani M. Ethylmalonic encephalopathy is caused by mutations in the ETHE1, a gene coding a mitochondrial matrix protein. Am J Hum Genet 2004; 74 : 239-252

11 Tiranti V, Briem E, Lamantea E, Mineri R, Papaleo E, De Gioia L, Forlani F, Rinaldo P, Dickson P, Abu-Libdeh B, Cindro-Heberle L, Owaidha M, Jack RM, Christensen E, Burlina A, Zeviani M. ETHE1 mutations are specific to ethylmalonic encephalopathy. J Med Genet 2006; 43: 340-346

12 Yoon HR, Hahn SH, Ahn YM, Jang SH, Shin YJ, Lee EH, Ryu KH, Eun BL, Rinaldo $P$, Yamaguchi $S$. Therapeutical trial in the first three Asian cases of ethylmalonic encephalopathy: response to riboflavine. J Inherit Metab Dis 2001; 24: 870-873

13 Zafeiriou DI, Augoustides-Savvapoulou P, Haas D, Smet J, Triantafyllou P, Vargiami E, Tamiolaki M, Gombakis N, Coster R van, Sewell AC, VianeySaban C, Gregersen N. Ethylmalonic encephalopathy: clinical and biochemical observations. Neuropediatrics 2007; 38: 78-82 